\title{
Diaphragmatic Eventration in Nf1 Microdeletion Syndrome: A Rare Association Unmasked by Pregnancy
}

\author{
Marchetti GB ${ }^{1}$, Cagnoli GA*1, Villa $\mathrm{R}^{1}$, Scuvera $\mathrm{G}^{2}$, Verga $\mathrm{U}^{3}$, Trespidi $\mathrm{L}^{4}$, Radaelli $\mathrm{T}^{4}$, Bianchessi \\ $D^{5}$, Palleschi $A^{6}$, Cesaretti $C^{1}$ and Natacci $F^{1}$ \\ ${ }^{1}$ Fondazione IRCCS Ca Granda Ospedale Maggiore Policlinico, Medical Genetics Unit, Woman-Child-Newborn Department, \\ Milan, Italy
}

${ }^{2}$ Fondazione IRCCS Ca Granda Ospedale Maggiore Policlinico, Pediatric Highly Intensive Care Unit, Woman Child-Newborn Department, Milan, Italy

${ }^{3}$ Fondazione IRCCS Ca Granda Ospedale Maggiore Policlinico Endocrinology Unit, Internal Medicine Department, Milan, Italy

${ }^{4}$ Fondazione IRCCS Ca Granda Ospedale Maggiore Policlinico Gynecology and Obstetrics Unit, Woman-Child-Newborn Department, Milan, Italy

${ }^{5}$ Fondazione IRCCS Istituto Neurologico Carlo Besta, Molecular Neuro-Oncology Unit, Milan, Italy

${ }^{6}$ Fondazione IRCCS Ca' Granda Ospedale Maggiore Policlinico, Thoracic Surgery and Lung Transplantation Unit, Surgery Department, Milan, Italy

*Corresponding author: Cagnoli Giulia Anna, Fondazione IRCCS Ca Granda Ospedale Maggiore Policlinico, Medical Genetics Unit, Woman-Child-Newborn Department, Milan, Italy

\section{ARTICLE INFO}

Received: 蔧 July 01, 2021

Published: 催 July 14, 2021

Citation: Marchetti GB, Cagnoli GA, Villa R, Scuvera G, Verga U. Diaphragmatic Eventration in Nf1 Microdeletion Syndrome: A Rare Association Unmasked by Pregnancy. Biomed J Sci \& Tech Res 37(2)2021. BJSTR. MS.ID.005964.

Keywords: NF1; Neurofibromatosis; Microdeletion; Diaphragmatic Defect; Eventration; Pregnancy Complications; Rare Disease

\section{ABSTRACT}

Background: Neurofibromatosis type 1 (NF1) is a cancer predisposing condition caused by mutations occurring in the NF1 gene, coding for Neurofibromin. Among mutations on NF1 gene, Type 1 microdeletions are those related to more severe manifestations. NF1 women may undergo several complications during pregnancy.

Case Presentation: We describe the unique case of a NF1 micro deleted woman in which pregnancy unmasked the presence of a diaphragmatic defect, likely congenital, and the multidisciplinary management of this association.

Conclusion: Diaphragmatic defects have never been described before in a NF1 micro deleted pregnant woman. In consideration of the rareness of this condition, the small number of cases reported in literature and the considerable number of comorbidities in these patients, it is mandatory that pregnant women affected by such a rare condition should be followed by a multidisciplinary specialistic clinical team.

Abbreviations: NF1: Neurofibromatosis Type 1; NIH: National Institutes of Health; CDD: Congenital Diaphragmatic Defects; DE: Diaphragm Eventration; CDE: Congenital DE; MRI: Magnetic Resonance Imaging; CT: Computed Tomography; VATS: Video-Assisted Thoracoscopic Surgery; MPNSTs: Malignant Peripheral Nerve Sheath Tumors

\section{Introduction}

Neurofibromatosis type 1 (NF1), also known as von Recklinghausen disease, is a neurocutaneous condition with an estimated prevalence between 1 in 2000 to 1 in 4000, characterized by a great variability in its clinical presentation. Clinical hallmarks such as café au-lait macules, neurofibromas, axillar or inguinal freckling, optic pathway glioma and iris hamartomas (Lisch nodules) 
represent the main NF1 diagnostic criteria set by the U.S. National Institutes of Health (NIH) in 1988 [1]. Beside these typical signs, the possible association with skeletal, endocrine, cardiovascular and oncologic complications make NF1 a multi-systemic disorder [2]. A similar broad spectrum of clinical manifestation is caused by mutations occurring in the Neurofibromin on co suppressor gene, on chromosome band 17q11.2, and inherited in an autosomic dominant manner [3]. In about $5-10 \%$ of cases takes place a large deletion involving the NF1 gene and its flanking regions which results in the "NF1 microdeletion syndrome" [4]. Despite the lack of genotype-phenotype correlations in NF1, patients with Type 1 microdeletion (1.4 Mb encompassing about 14 genes) typically present a serious illness, mainly characterized by dysmorphic facial features and developmental delay [5]. During pregnancy, NF1 women should be monitored because they are more prone to complications such as hypertension, preeclampsia, fetopelvic disproportion, poor fetal growth and oligohydramnios [6]. Furthermore, pregnancy for NF1 women is related to an increased number and size of cutaneous neurofibromas [7]. Regarding pregnancy in women with NF1 microdeletion, no specific literature data are available, possibly due to their low fitness and to the rarity of the condition.

Diaphragmatic defects have never been described before as a pregnancy complication in NF1 women. Similar conditions are also unusual in general population, with only 56 reported cases until 2018 [8]. The increased abdominal pressure experienced by women during pregnancy might represent a risk factor for unmasking hidden congenital diaphragmatic defects (CDD). Among these defects, diaphragm eventration (DE) represents an uncommon finding, defined as the upward displacement of a portion or the entire diaphragm, otherwise intact. Congenital DE (CDE) diagnosis is often an incidental finding in adults, as it can remain completely asymptomatic. Even if scarcely described first presentation of CDD during pregnancy, owing to the risk of rupture, represents a lifethreatening complication both for mother and fetus [9]. Here we report a case of (CDE) complicating the pregnancy of a 30-year-old patient with NF1 Type 1 Microdeletion Syndrome.

\section{Case Report}

The patient is a 30-year-old pregnant woman, affected by NF1 type 1 microdeletion syndrome, followed since the age of 24 at our Clinical Reference Centre for NF1. She has undergone numerous clinical and instrumental evaluations over the years; the main clinical issues that have emerged are summarized here: mild intellectual disability, typical NF1 cutaneous features (cafè au laits spots and diffuse cutaneous neurofibromas) and two plexiform neurofibromas on the scalp and on the left hemiabdomen. Furthermore, multiple spinal neurofibromas were reported at the age of 26 , involving all the conjugation foramina, especially in the thoracic and lumbar tracts of the spine. At the age of 28 due to a suspected diagnosis of pheochromocytoma, the patient underwent left adrenalectomy; histological examination diagnosed a ganglioneuroma and steroid replacement therapy was therefore introduced. In addition, a nonfunctioning pituitary microadenoma was detected on routine brain magnetic resonance imaging (MRI), steady in size on control MRI in the following years. In view of these comorbidities, as the patient got pregnant, she was followed by a multidisciplinary team, consisting of gynecologists, endocrinologists, neurologists and medical geneticists. Regular ultrasound evaluations were performed throughout the entire pregnancy. The patient and her partner decided not to proceed with any invasive tests for prenatal diagnosis.

The pregnancy progressed regularly. In preparation of spinal anesthesia, a spine MRI was performed to monitor the wellknown spinal neurofibromas that appeared to be steady in size and number. Serendipitously, three round-shaped images at the base of right chest were reported and interpreted as possible diaphragmatic hernia (DH) with partial displacement of the liver to the chest. A right-sided pleural effusion was also reported, which retrospectively appeared to be present and unchanged since the previous control. An echocardiogram and an abdominal ultrasound were performed as follow-up investigations. The former reported mild mitral and tricuspid insufficiency, but no pericardial effusion was observed. The second examination showed a moderate pleural effusion on the right side with thin fibrinous septae and gross outpouchings of the liver parenchyma, the largest of almost $4 \mathrm{~cm}$. Thus, the previous suspicion of DH was confirmed. In view of the numerous comorbilities, a caesarean section was performed and the patient gave birth to a female newborn. Genetic NF1 analysis performed after birth revealed the transmission of the maternal NF1 microdeletion to the baby. Since both the mother and the newborn were in good health, they were discharged five days after delivery, once DH-related complications were excluded. Computed Tomography (CT) of the upper abdomen performed two months later showed three diaphragmatic bulges, with a maximum diameter of $4.2 \mathrm{~cm}$ on the VIII segment of the liver and of $4.8-2.8 \mathrm{~cm}$ on the VII segment. The right-sided pleural effusion was still present and, as the diaphragm was not clearly visible over hepatic protrusions and appeared worsen from previous imaging, the hypothesis of hernias was corroborated (Figure 1). Therefore, surgical correction of the defect was planned after the weaning of the child. The patient underwent video-assisted thoracoscopic surgery (VATS), which revealed an intact but very thin diaphragm, consistent with a diagnosis of DE. There was no evidence of occult neurofibromas nearby, nor adjacent lesions that might be the very first cause of the diaphragmatic eventration. In addition, a voluminous pleuro-pericardial cyst was found, explaining the pleural effusion previously reported. Finally, a diaphragm plication and cyst excision were performed. The removed tissue was biopsied and this examination revealed the presence of a cystic wall, covered by a single stratified cubic-cylindrical epithelium. No atypia was found. The post-operative course was uneventful. 

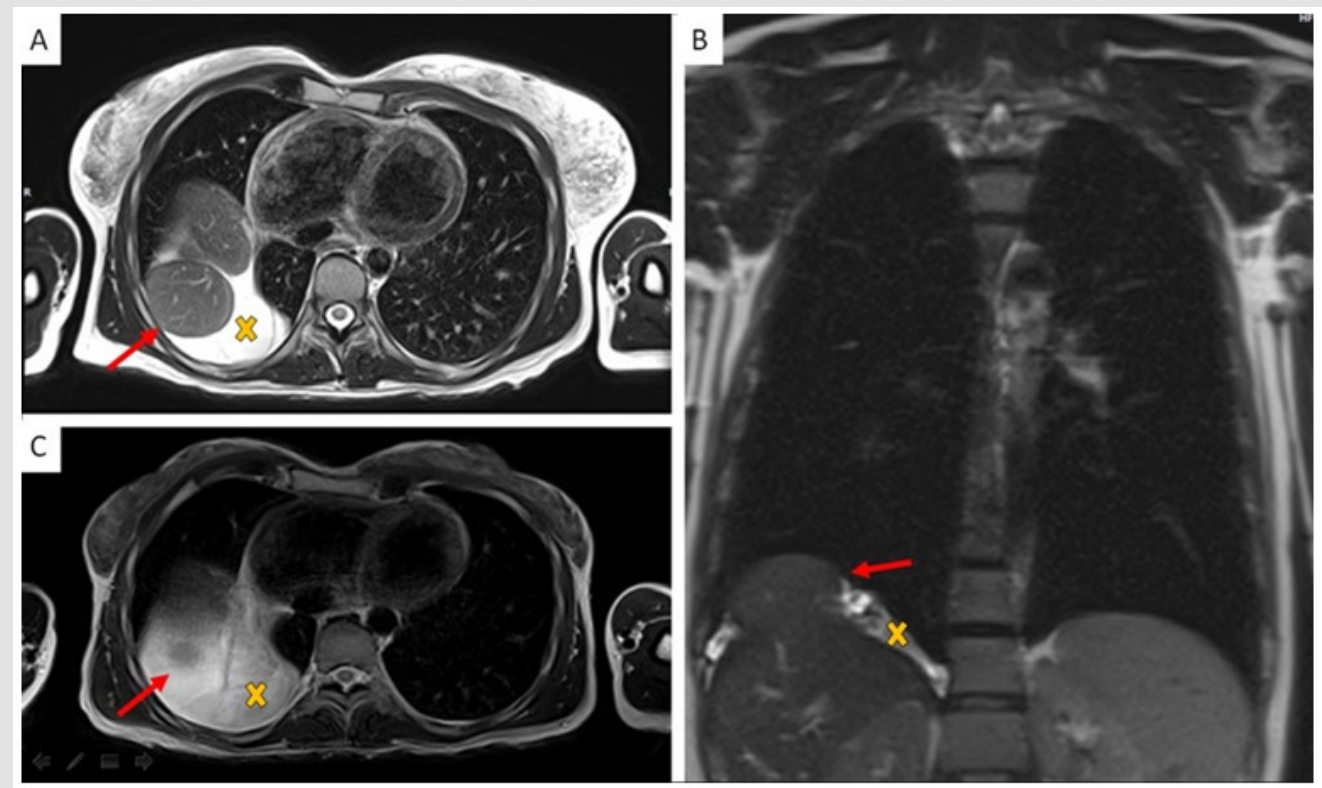

Figure 1: Chest MR Imaging:

A. Two round shape images with partial liver shift in right pleural space (red arrow) and surrounding fluid collection (yellow X);

B. Coronal plane;

C. MR imaging three years before pregnancy.

\section{Discussion}

Diaphragmatic weakness became clinically relevant during pregnancy, when the rising abdominal pressure pushes the diaphragm upwards while the muscle itself contracts downwards. In our patient, these two opposing forces probably enlarged a preexisting diaphragmatic defect, resulting in liver herniation. To our knowledge this is the first reported case of a patient with NF1 microdeletion showing a DE and a pericardial cyst, three extremely rare conditions. Fortunately, the liver was the only organ involved in herniation and the patient remained asymptomatic, even during pregnancy. As mentioned, among NF1 population, patients with type 1 microdeletion generally display a more severe phenotype. This is possibly due to the deletion not only of the NF1 gene, but also of its flanking genes, which could partially influence the clinical manifestation of the disease. In particular, NF1 type 1 microdeleted patients are more likely to develop malignant peripheral nerve sheath tumors (MPNSTs), cardiovascular anomalies [5] and connective tissue abnormalities [10]. Although the precise molecular basis of connective tissue involvement in NF1 is still unclear, neurofibromin has been shown to play a regulatory role in mesenchymal stem cell differentiation [10]. Moreover, during embryogenesis, this protein also takes part to axons' elongation in order to ensure the correct activity of the nervous system [11]. The involvement of NF1 gene in proper neuronal and mesenchymal development must be particularly stressed as neurons and connective tissue, together with several other structures from different embryonic origins, participate to the complex sequence of events that leads to diaphragm development [12]. In particular, CDE has been related to defects in migration and proliferation of muscle fibres, two steps leaning on the regulatory action of connective tissue cells [13].

Whether the association between NF1 and CDE is causal or is in fact a possible associated complication, the management of our case demonstrates the crucial importance of a multidisciplinary approach to pregnant women affected by a rare disease. NF1 microdeletion syndrome is associated with numerous comorbidities, most of which are just barely known. Particularly, knowledge about pregnancy-related complications in NF1 microdeleted women is still lacking and even more about possible urgent events; as a consequence, no specific management guidelines are available yet. As shown by our case report, pregnancy in these women can turn out to be a challenging moment and should be carefully supervised, as it represents an event in which the delicate equilibrium of such fragile patients might unbalance, possibly leading to unexpected, unknown and potentially serious complications. In our opinion the complexity of this condition, the unpredictability of complications' onset and severity can be properly managed only by gaining a wider and multifaceted vision of the disease, which can be obtained only by means of a dedicated multidisciplinary team. A well-coordinated multi-specialized equipe could represent the primary step 
towards widening our perspective of the condition and towards implementing tools for personalized care and follow-up, with the ultimate aim of ensuring early diagnosis and prompt management of possible upcoming emergencies, especially during pregnancy in these vulnerable women.

\section{Disclosure of Potential Conflicts of Interest}

No potential conflict of interest was disclosed.

\section{References}

1. (1988) Neurofibromatosis Conference statement. National Institutes of Health Consensus Development Conference. Arch Neurol 45(5): 575578.

2. Ferner RE, Huson SM, Thomas N, Moss C, Willshaw H, et al. (2007) Guidelines for the diagnosis and management of individuals with neurofibromatosis 1. J Med Genet 44(2): 81-88.

3. Friedman JM (2020) Neurofibromatosis 1. In: Adam MP, Ardinger HH, Pagon RA, Wallace SE, Bean LJH, Stephens K, Amemiya A (Eds.)., GeneReviews ${ }^{\circledR}$ Seattle (WA): University of Washington, Seattle; 19932020. PMID: 20301288.

4. Brussa Reis L, Turchetto Zolet AC, Fonini M, Ashton Prolla P, Rosset C (2019) The Role of Co-Deleted Genes in Neurofibromatosis Type 1 Microdeletions: An Evolutive Approach. Genes (Basel) 10(11): 839.

5. Kehrer Sawatzki H, Mautner VF, Cooper DN (2017) Emerging genotypephenotype relationships in patients with large NF1 deletions. Hum Genet 136(4): 349-376.

\section{ISSN: 2574-1241}

DOI: 10.26717/BJSTR.2021.37.005965

Cagnoli GA. Biomed J Sci \& Tech Res

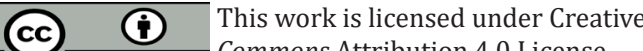
Commons Attribution 4.0 License

Submission Link: https://biomedres.us/submit-manuscript.php
6. Leppävirta J, Kallionpää RA, Uusitalo E, Vahlberg T, Pöyhönen M, et al. (2017) The pregnancy in neurofibromatosis 1: A retrospective registerbased total population study. Am J Med Genet A 173(10): 2641-2648.

7. Cesaretti C, Melloni G, Quagliarini D, Fogliani R, Zaina B, et al. (2013) Neurofibromatosis type 1 and pregnancy: maternal complications and attitudes about prenatal diagnosis. Am J Med Genet A 161A(2): 386-388.

8. Reddy M, Kroushev A, Palmer K (2018) Undiagnosed maternal diaphragmatic hernia - a management dilemma. BMC Pregnancy Childbirth 18(1): 237.

9. Servais EL, Stiles BM, Finnerty BM, Paul S (2012) Ruptured diaphragmatic eventration: a rare cause of acute postpartum dyspnea. Ann Thorac Surg 93(6): e143-144.

10. Mensink KA, Ketterling RP, Flynn HC, Knudson RA, Lindor NM, et al. (2006) Connective tissue dysplasia in five new patients with NF1 microdeletions: further expansion of phenotype and review of the literature. J Med Genet 43(2): e8.

11. Larizza L, Gervasini C, Natacci F, Riva P (2009) Developmental abnormalities and cancer predisposition in neurofibromatosis type 1 . Curr Mol Med 9(5): 634-653.

12. Sefton EM, Gallardo M, Kardon G (2018) Developmental origin and morphogenesis of the diaphragm, an essential mammalian muscle. Dev Biol 440(2): 64-73.

13. Merrell AJ, Ellis BJ, Fox ZD, Lawson JA, Weiss JA, et al. (2015) Muscle connective tissue controls development of the diaphragm and is a source of congenital diaphragmatic hernias. Nat Genet 47(5): 496-504.

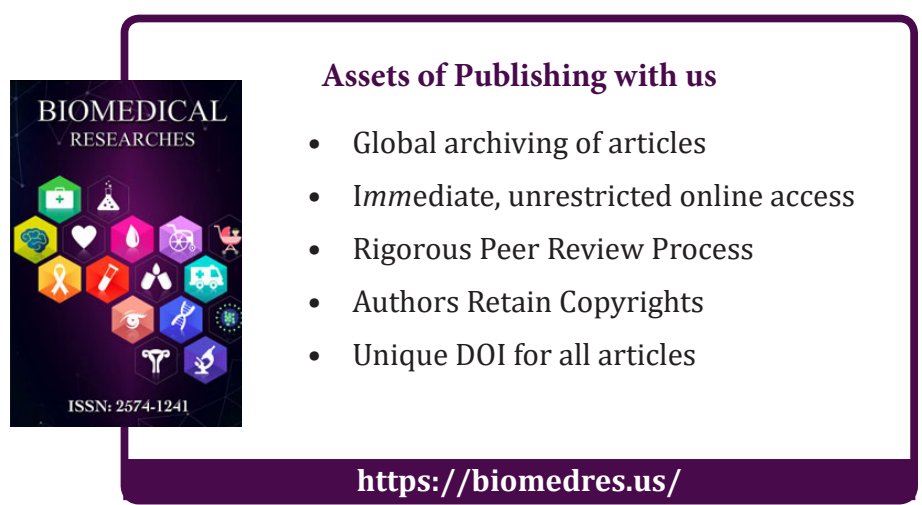

